\title{
Combined immune checkpoint inhibitor and chemotherapy is effective in a patient with ALK rearranged non-small cell lung cancer: a case report
}

\author{
Lexin $\mathrm{Xia}^{1}$, Hanguang $\mathrm{Hu}^{2}$, Wen $\mathrm{Li}^{1}$, Huahao Shen ${ }^{1}$, Yang $\mathrm{Xia}^{1}$ \\ ${ }^{1}$ Department of Respiratory and Critical Care Medicine, ${ }^{2}$ Department of Medical Oncology, Second Affiliated Hospital of Zhejiang University \\ School of Medicine, Hangzhou 310009, China \\ Correspondence to: Yang Xia, MD, PhD. Department of Respiratory and Critical Care Medicine, Second Affiliated Hospital, Zhejiang University \\ School of Medicine, Hangzhou 310009, China. Email: yxia@zju.edu.cn.
}

\begin{abstract}
Targeted therapies are validated to be efficient in non-small cell lung cancer (NSCLC) patients with driver gene mutations, but the emergence and development of immune checkpoint inhibitors (ICIs) in the last decades offers new insight into the therapeutic decisions of patients harboring driver gene mutations. The appropriate application of ICIs-based strategies in these patients remains to be assessed. Here, we present a patient with chest tightness and nonproductive cough. A primary site was found in left lower lobe with pleural effusion, pericardium effusion and multiple enlarged lymph nodes. He was diagnosed with advanced NSCLC and responded well to the first line pembrolizumab combined with pemetrexed and carboplatin with a progression free survival of 26 months. Immunohistochemistry analysis of relapsed 4R lymph node indicated ALK-positive. Surprisingly, identical form of ALK gene rearrangement was observed in re-biopsy sample and the specimen 2 years ago. The patient was again dramatically benefit from 2L alectinib. No adverse event was presented and the progression-free survival is not reached until this case report. Hence, the current case suggested that combined immunotherapy and chemotherapy might be an efficient and tolerable alternative at $1 \mathrm{~L}$ or after resistance to standard of care in patients with oncogene mutant NSCLC. Further data is required to make this a practical ambition.
\end{abstract}

Keywords: Anaplastic lymphoma kinase; chemotherapy; immunotherapy; non-small cell lung cancer (NSCLC); case report

Submitted Jul 26, 2019. Accepted for publication Dec 03, 2019.

doi: $10.21037 /$ tcr.2019.12.39

View this article at: http://dx.doi.org/10.21037/tcr.2019.12.39

\section{Introduction}

Lung cancer is the most commonly diagnosed cancer and the leading cause of cancer death worldwide (1). Non-small cell lung cancer (NSCLC) approximately accounts for $85 \%$ of all lung cancers, while adenocarcinoma constitutes $70 \%$ to $80 \%$ of NSCLC (2). Targeted therapy provides patients with enormous survival benefit and is the first-line therapy for advanced NSCLC with driver gene mutations. However, in recent years, not only did immunotherapy emerge and develop, but it also greatly challenged the status of targeted therapy. A lot of questions remain to be answered about whether immunotherapy should be applied alone or in combination, with chemotherapy or tyrosine kinase inhibitors, first line or later lines, and how to screen the suitable candidates for ICI treatment. Here we presented a patient with NSCLC receiving first line pembrolizumab in combination with chemotherapy and gained two years of progression free survival (PFS) with ALK rearrangement. This case provided evidence that immunotherapy plus chemotherapy might be an efficient and tolerable choice at $1 \mathrm{~L}$ or after resistance to standard of care in NSCLC patients with ALK fusion. We present the following case in accordance with the CARE Guideline. 


\section{Case presentation}

A 56-year-old male with a smoking history of 60 packyears was admitted for chest tightness and nonproductive cough. Enhanced chest computed tomography and positron emission tomography-computed tomography scan showed suspicious primary site in left lower lobe, bilateral pleural effusion and pericardium effusion, with enlarged mediastinal, hilar, supraclavicular and cervical lymph nodes. No medical or family history of lung cancer were presented. Exfoliated cells from pleural effusion suggested lung adenocarcinoma, cT2N3M1a, stage IV A, and EGFR, ALK, ROS-1 and MET by amplification refractory mutation system were all wildtype. He underwent chemotherapy (pemetrexed $800 \mathrm{mg}$ D1, carboplatin $600 \mathrm{mg}$ D1) combined with pembrolizumab (100 mg, D1). The malignant pleural and pericardium effusions were well-controlled, and the lung was recruited, leaving a fusiform lesion in the left lower lobe at 6-week response evaluation. This residual lesion dramatically regressed when treated with additional 4 cycles of pemetrexed $800 \mathrm{mg}$ D1, carboplatin $600 \mathrm{mg}$ D1 plus pembrolizumab $100 \mathrm{mg} \mathrm{D1}$, and kept shrinking during maintenance therapy of pemetrexed $800 \mathrm{mg}$ D1 and pembrolizumab $100 \mathrm{mg}$ D1 in the subsequent 2 years. However, increasing plasma carcinoembryonic antigen was substantially monitored since 16-month maintenance therapy with no radiographic progression. An enlarged 4R lymph node was identified afterward and confirmed by endobronchial ultrasound-guided transbronchial needle aspiration and immunohistochemistry (IHC) analysis as ALK-positive metastatic lung adenocarcinoma. Additional IHC analysis was conducted on the paraffin-embedded specimen and revealed ALK positive. In extension, we carried out nextgeneration sequencing using re-biopsy sample together with the specimen 2 years ago and the results suggested the identical form of ALK gene rearrangement. 2L using alectinib was given. The enlarged lymph node disappeared, and the plasma CEA fell to the normal range after 2-month treatment (Figures 1,2). Alectinib was well-tolerated at 2L and to date, the progression-free survival is not reached.

\section{Discussion}

Immune checkpoint inhibitors (ICIs) are a new standard of care for patients with advanced non-small-cell lung cancer (NSCLC), however, the role of immunotherapy in patients with ALK positive NSCLC is not clear. 1L chemotherapy was effective in advanced ALK-positive NSCLC with a progression-free survival (PFS) of 7.0 months and an objective response rate of $45 \%$ (3). In our case, the patient was given chemotherapy plus pembrolizumab with 26 months of PFS, which far exceeded the conventional PFS of chemotherapy. The IMpower 150 study enrolled patients with metastatic nonsquamous NSCLC and observed an improved median PFS and overall survival (OS) when treated with atezolizumab plus chemotherapy and bevacizumab (4). Prolonged mPFS and mOS was observed in patients with EGFR/ALK alterations given extra atezolizumab on top of chemotherapy and bevacizumab compared to chemotherapy plus bevacizumab alone (PFS 9.7 vs. 6.1 months, HR 0.59, 95\% CI: 0.37-0.94, OS 22.5 vs. 16.4 months, HR 0.77; 95\% CI: 0.58-1.04) (4). These data suggest that ICIs combined with chemotherapy might serve as an alternative strategy for EGFR/ALK altered patients. Of note, our case relapsed at mediastinal lymph node with confirmed identical ALK-fusion despite substantial maintenance therapy, suggesting ICI plus chemotherapy though effective, still failed to eliminate oncogene-positive tumor subclone. PD-L1 expression and tumor mutation burden was not taken into analysis, which is a major limitation for investigating the underlying mechanism.

Hence, the question remains to leave open that what is the optional ICIs-based combination of treating EGFR/ ALK aberrant population? Anti-EGFR/ALK agents are standard treatments for patients with EGFR/ALK mutant advanced NSCLC. Nivolumab plus crizotinib causes severe hepatic toxicities leading to the discontinuation of the combination in over $1 / 3$ patients (5). In contrast, a sequential combined therapy of chemotherapy and crizotinib followed by ICIs leads to elevated $\mathrm{T}$ cell infiltration and increased antitumor effects (6). On the other hand, osimertinib or gefitinib plus $\mathrm{PD}(\mathrm{L})-1$ blockage instigated intolerable toxicity. Pembrolizumab plus erlotinib although is feasible in terms of toxicity, failed to improve the therapeutic efficiency. Additionally, ICIs followed by osimertinib was associated with severe immune-related adverse events (7).

Collectively, combined immunotherapy and chemotherapy is an efficient and tolerable alternative at $1 \mathrm{~L}$ or after resistance to standard of care in patients with oncogene mutant NSCLC. Further data is required to make this a practical ambition. 

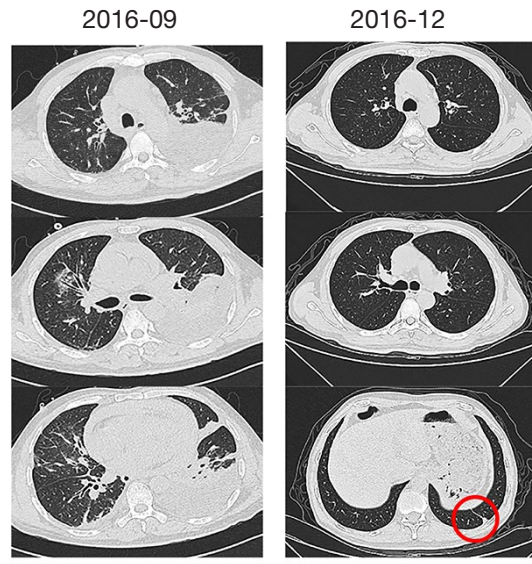

2017-02

2016.10-2017.02 pembrolizumab + pemetrexed + carboplatin $\times 6$ cycles

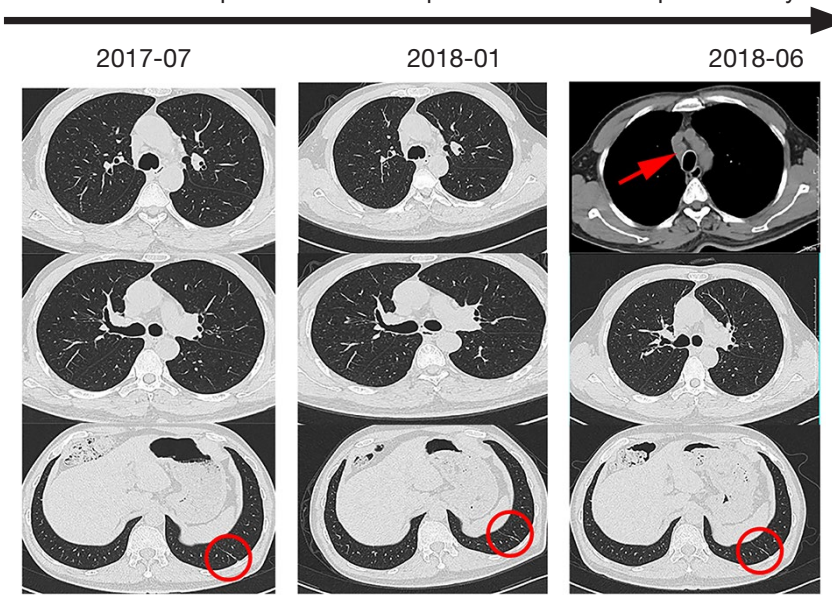

2017.02-2018.11 pembrolizumab + pemetrexed $\times 16$ cycles

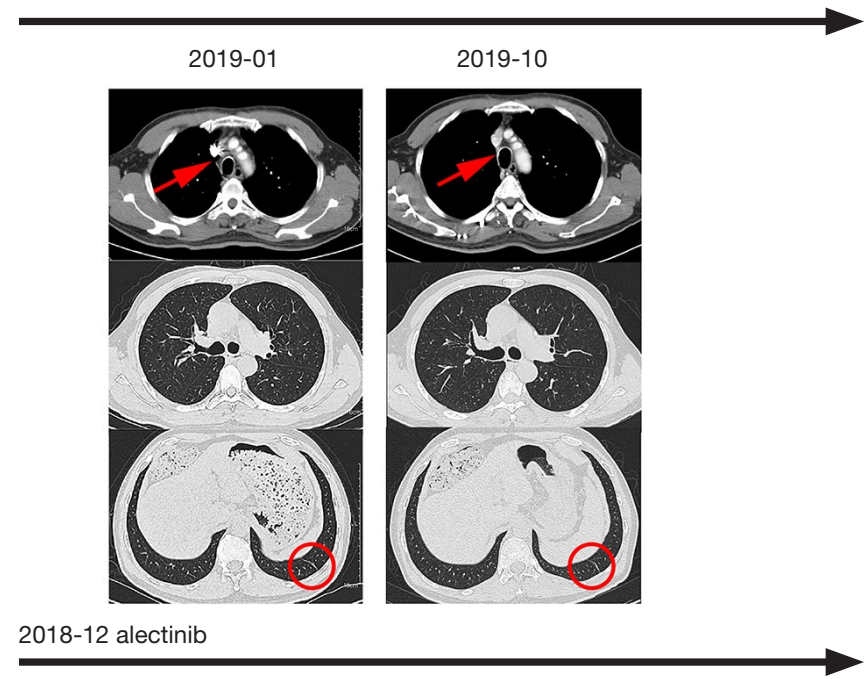

Figure 1 CT manifestation during treatment. Chest CT showed primary site in left lower lobe, bilateral pleural effusion, pericardium effusion and enlarged mediastinal lymph node. After treated with six cycles of pemetrexed and carboplatin combined with pembrolizumab, the malignant pleural and pericardium effusions were well-controlled, and the residual lesion kept shrinking for two years during maintenance therapy of pemetrexed and pembrolizumab. An enlarged 4R lymph node was identified and disappeared after 2-month alectinib treatment. The circles indicate the suspected primary site on the left lower lobe. CT, computed tomography. 


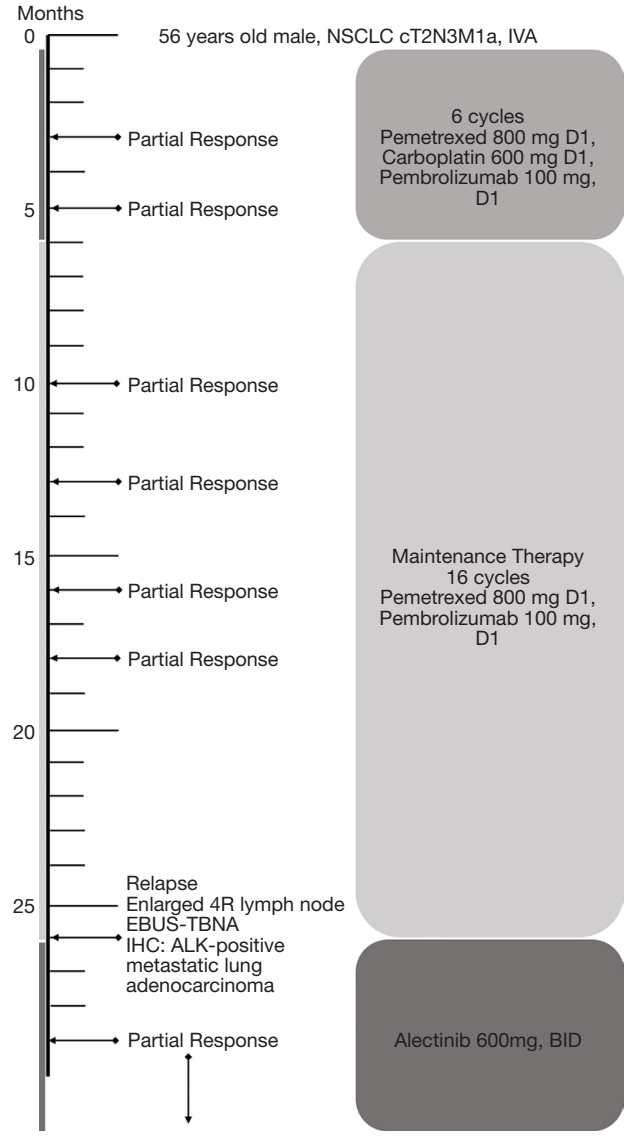

Figure 2 Timeline for the episode of care.

\section{Acknowledgments}

Funding: This work was supported by the Zhejiang Provincial Natural Science Foundation of China (LY20H010004), the National Natural Science Foundation of China (81870022) and the Medical and Health Technology Program of Zhejiang Province (2017204226).

\section{Footnote}

Conflicts of Interest: All authors have completed the ICMJE uniform disclosure form (available at http://dx.doi. org/10.21037/tcr.2019.12.39). The authors have no conflicts of interest to declare.

Ethical Statement: The authors are accountable for all aspects of the work in ensuring that questions related to the accuracy or integrity of any part of the work are appropriately investigated and resolved. All procedures performed in studies involving human participants were in accordance with the Declaration of Helsinki (as revised in 2013). Written informed consent was obtained from the patient for publication of this manuscript and any accompanying images.

Open Access Statement: This is an Open Access article distributed in accordance with the Creative Commons Attribution-NonCommercial-NoDerivs 4.0 International License (CC BY-NC-ND 4.0), which permits the noncommercial replication and distribution of the article with the strict proviso that no changes or edits are made and the original work is properly cited (including links to both the formal publication through the relevant DOI and the license). See: https://creativecommons.org/licenses/by-nc-nd/4.0/.

\section{References}

1. Bray F, Ferlay J, Soerjomataram I, et al. Global cancer statistics 2018: GLOBOCAN estimates of incidence and mortality worldwide for 36 cancers in 185 countries. CA Cancer J Clin 2018;68:394-424.

2. Molina JR, Yang P, Cassivi SD, et al. Non-small cell lung cancer: epidemiology, risk factors, treatment, and survivorship. Mayo Clin Proc 2008;83:584-94.

3. Solomon BJ, Mok T, Kim DW, et al. First-line crizotinib versus chemotherapy in ALK-positive lung cancer. N Engl J Med 2014;371:2167-77.

4. Socinski MA, Jotte RM, Cappuzzo F, et al. Overall survival (OS) analysis of IMpower150, a randomized $\mathrm{Ph} 3$ study of atezolizumab (atezo) plus chemotherapy (chemo) +/bevacizumab (bev) vs chemo + bev in 1L nonsquamous (NSQ) NSCLC. J Clin Oncol 2018;36:9002.

5. Spigel DR, Reynolds C, Waterhouse D, et al. Phase $1 / 2$ Study of the Safety and Tolerability of Nivolumab Plus Crizotinib for the First-Line Treatment of Anaplastic Lymphoma Kinase Translocation - Positive Advanced Non-Small Cell Lung Cancer (CheckMate 370). J Thorac Oncol 2018;13:682-8.

6. Liu P, Zhao L, Pol J, et al. Crizotinib-induced immunogenic cell death in non-small cell lung cancer. Nat Commun 2019;10:1486.

7. Schoenfeld AJ, Arbour KC, Rizvi H, et al. Severe immunerelated adverse events are common with sequential PD-(L)1 blockade and osimertinib. Ann Oncol 2019;30:839-44.

Cite this article as: $\mathrm{Xia} \mathrm{L,} \mathrm{Hu} \mathrm{H,} \mathrm{Li} \mathrm{W,} \mathrm{Shen} \mathrm{H,} \mathrm{Xia} \mathrm{Y.}$ Combined immune checkpoint inhibitor and chemotherapy is effective in a patient with ALK rearranged non-small cell lung cancer: a case report. Transl Cancer Res 2020;9(3):2045-2048. doi: $10.21037 /$ tcr.2019.12.39 